\title{
Pelvic nerve injury causes a rapid decrease in expression of choline acetyltransferase and upregulation of c-Jun and ATF-3 in a distinct population of sacral preganglionic neurons
}

\author{
Christopher J. Peddie and Janet R. Keast* \\ Pain Management Research Institute and Kolling Institute of Medical Research, University of Sydney at Royal North Shore Hospital, St Leonards, NSW, Australia
}

Edited by:

Margaret A. Vizzard, University of

Vermont College of Medicine, USA

Reviewed by:

Meredith T. Robbins, University of

Alabama at Birmingham, USA

Vladimir Zagorodnyuk, Flinders

University, Australia

${ }^{*}$ Correspondence:

Janet R. Keast, Royal North Shore

Hospital, Kolling Building (Level 13),

St Leonards, NSW 2065, Australia.

e-mail: janet.keast@sydney.edu.au
Autonomic regulation of the urogenital organs is impaired by injuries sustained during pelvic surgery or compression of lumbosacral spinal nerves (e.g., cauda equina syndrome). To understand the impact of injury on both sympathetic and parasympathetic components of this nerve supply, we performed an experimental surgical and immunohistochemical study on adult male rats, where the structure of this complex part of the nervous system has been well defined. We performed unilateral transection of pelvic or hypogastric nerves and analyzed relevant regions of lumbar and sacral spinal cord, up to 4 weeks after injury. Expression of c-Jun, the neuronal injury marker activating transcription factor-3 (ATF-3), and choline acetyltransferase (ChAT) were examined. We found little evidence for chemical or structural changes in substantial numbers of functionally related but uninjured spinal neurons (e.g., in sacral preganglionic neurons after hypogastric nerve injury), failing to support the concept of compensatory events. The effects of injury were greatest in sacral cord, ipsilateral to pelvic nerve transection. Here, around half of all preganglionic neurons expressed c-Jun within 1 week of injury, and substantial ATF-3 expression also occurred, especially in neurons with complete loss of ChAT-immunoreactivity. There did not appear to be any death of retrogradely labeled neurons, in contrast to axotomy studies performed on other regions of spinal cord or sacral ventral root avulsion models. Each of the effects we observed occurred in only a subpopulation of preganglionic neurons at that spinal level, raising the possibility that distinct functional subgroups have different susceptibility to trauma-induced degeneration and potentially different regenerative abilities. Identification of the cellular basis of these differences may provide insights into organ-specific strategies for attenuating degeneration or promoting regeneration of these circuits after trauma.

Keywords: spinal cord injury, spinal nerves, cauda equina syndrome, inferior hypogastric plexus, micturition, regeneration, sprouting

\section{INTRODUCTION}

Parasympathetic preganglionic neurons in the sacral spinal cord are essential for urogenital reflexes, including voiding (micturition and defecation), penile erection, and stimulation of secretions from the internal reproductive tract. Their axons project via the spinal and then the pelvic nerves, therefore damage to the sacral cord or these nerves causes major problems with urogenital control that significantly impact on the health and quality of life (Fowler, 1999; Orendacova et al., 2001; Podnar et al., 2006; Samson and Cardenas, 2007). Sacral preganglionic neurons release acetylcholine to excite motor neurons in the pelvic ganglia, also known as the pelvic plexus or, in humans, the inferior hypogastric plexus (Keast, 1999). A separate population of pelvic ganglion neurons receive excitatory cholinergic inputs from sympathetic preganglionic neurons in the upper lumbar cord that project in the lumbar spinal nerves and then the hypogastric nerve (Tabatai et al., 1986; Keast, 1995). Therefore, this mixed autonomic ganglion contains highly targeted excitatory inputs from spatially distinct regions of the spinal cord.

Following injury to either the pelvic or hypogastric nerves, the complexity of pelvic autonomic circuitry leads to a number of very interesting events that cannot be explained simply by a subtraction of one group of inputs to the pelvic ganglia and are more consistent with plasticity of spared nerves. For example, functional data suggests that following pelvic nerve transection, uninjured lumbar preganglionic axons grow collaterals to restore central innervation to parasympathetic pelvic ganglion neurons that mediate penile erection (Dail et al., 1989) and bladder contraction (de Groat and Kawatani, 1989); conversely, after hypogastric nerve injury, pelvic nerve activation causes contraction of the vas deferens, an outcome originally mediated by sympathetic spinal nerves (Kihara et al., 1996). There is also evidence for axon collateral growth within pelvic ganglia following pelvic or hypogastric nerve injury (Dail and Evan, 1978; Dail et al., 1997; Kepper and Keast, 1998). These collaterals establish aberrant, long-lasting local connections that may impair restoration of normal circuitry when preganglionic axons regenerate (Kepper and Keast, 1998; Keast, 2004). The growth of collaterals is associated with upregulation of the immediate early gene, c-Jun (Nangle and Keast, 2009), commonly implicated in injury and regenerative growth (Raivich et al., 2004), even though in this particular case neurons have not been injured. Therefore, c-Jun may provide a marker not only for axotomized neurons but also, in some circumstances, for sprouting of uninjured neurons. 
The broad aim of our study was to examine how axotomy affects sacral preganglionic neurons in adult rats during the period when peripheral targets of these neurons are undergoing considerable change (1-4 weeks following injury). Specifically, we have monitored neurotransmitter synthesis [choline acetyltransferase (ChAT) expression] and structure (soma size) to determine if the spinal neurons are likely to retain their normal properties after injury. We have also investigated immediate early gene expression [c-Jun and activating transcription factor-3 (ATF-3)] to determine if these are upregulated similarly to elsewhere in the injured nervous system (Herdegen et al., 1997; Herdegen and Leah, 1998; Kenney and Kocsis, 1998; Hai et al., 1999; Raivich, 2008). Moreover, given the upregulation of c-Jun in sprouting, uninjured pelvic ganglion neurons (Nangle and Keast, 2009), we considered that this strategy may also reveal sprouting, uninjured preganglionic neurons (e.g., in the lumbar spinal cord following sacral nerve injury, or vice versa). We chose a unilateral, complete injury (transection of pelvic or hypogastric nerves) to focus on changes that are more likely to be directly related to injury than regeneration, which we prevented by removal of a segment of nerve at the time of its transection. Our experiments were performed in male Wistar rats, in which the neuroanatomy of pelvic autonomic circuits has been well defined and the pelvic autonomic ganglion neurons located in large, paired major pelvic ganglia rather than a multi-ganglionated plexus (Purinton et al., 1973; Keast, 1999). Numerous changes in structure and gene expression occurred rapidly after injury, some of which were sustained for 4 weeks but, surprisingly, these were found in only a minority of injured preganglionic neurons. This heterogeneity of response to injury may point to variable ability of different types of spinal pathways to undergo regeneration or respond to pro-regenerative therapies.

\section{MATERIALS AND METHODS SURGICAL PROCEDURES}

All procedures were approved by the University of Sydney and Royal North Shore Hospital animal ethics committees, as required by the Australian Code of Practice for the Care and Use of Animals for Scientific Purposes (National Health and Medical Research Council of Australia). Fifty-four adult male Wistar rats (9-12 weeks of age) were used for these experiments, divided into three experimental groups (neuronal counts, intensity analysis, soma size analysis); 23 for hypogastric nerve transection, 23 for pelvic nerve transection, and 8 as naïve controls. Animals were purchased from the Animal Resources Centre (Murdoch, WA, Australia), and were housed in groups of three, with environmental enrichment, under a 12-h light-dark cycle with free access to food and water.

Seven days prior to nerve transection, each animal received an intraperitoneal injection of approximately $300 \mu \mathrm{l}$ of a $0.5 \%$ solution of FluoroGold (FG; Fluorochrome, Englewood, CO, USA) in sterile saline. FG is a retrograde neuronal tracer used here to reliably label sympathetic and parasympathetic preganglionic neurons that project from the spinal cord into the abdomen (Anderson and Edwards, 1994).

Surgical procedures were conducted under isoflurane anesthesia ( $4 \%$ for induction, and $1.8-2 \%$ for maintenance, in $\mathrm{O}_{2}$ ). To study the effects of axotomy on preganglionic neurons, the hypogastric or pelvic nerves were unilaterally transected a short distance from the major pelvic ganglion, and a 1-2 mm segment of nerve was removed in each case to prevent regeneration. In each animal, the contralateral side was sham operated using the same process to visualize the pelvic ganglion and hypogastric or pelvic nerves, but without nerve transection.

\section{TISSUE PREPARATION}

After performing nerve transection, the animals were allowed to recover for either 7,14 , or 28 days before being reanesthetized with sodium pentobarbitone $(80-100 \mathrm{mg} / \mathrm{kg}$, i.p.) and intracardially perfused with approximately $100 \mathrm{ml}$ of a saline solution (containing 1\% sodium nitrite and $5000 \mathrm{IU} / \mathrm{ml}$ heparin), followed by approximately $500 \mathrm{ml}$ of $4 \%$ paraformaldehyde in $0.1 \mathrm{M}$ phosphate buffer $(\mathrm{PB})$, $\mathrm{pH} 7.4$ and overnight fixation in the same fixative. The dissected cord was then washed in $0.1 \mathrm{M}$ phosphate-buffered saline (PBS), $\mathrm{pH} 7.2$, and divided into segments containing the full extent of the lumbar L1-L2, and sacral L6-S1 regions. The spinal cord segments were marked to differentiate ipsilateral and contralateral sides and incubated in 30\% sucrose/0.1 M PBS overnight before embedding in an inert mounting medium (OCT; Tissue-Tek, Sakura, Torrance, CA, USA) and cryosectioning horizontally at a thickness of $40 \mu \mathrm{m}$. Free-floating sections from a 1 in 4 series were subsequently processed for immunohistochemistry.

\section{IMMUNOHISTOCHEMISTRY}

For fluorescent double-labeling, free-floating sections were first incubated in $0.1 \mathrm{MPBS}$ pH 7.2 containing $10 \%$ normal horse serum (NHS). Sections were then incubated for $48-72 \mathrm{~h}$ at room temperature with the following primary antisera combinations (antibody properties described below): ATF-3 (1:500) with ChAT (1:500); or ChAT with FG (1:2000). Each antibody combination was made up in PBS containing $0.1 \%$ sodium azide, $2 \%$ NHS, and $0.5 \%$ Triton $\mathrm{X}-100$. After washes in PBS, sections were subsequently incubated for $4 \mathrm{~h}$ at room temperature with donkey anti-rabbit Cy3 (1:1500) and donkey anti-sheep AF488 (1:1000). After further washes in PBS, sections were mounted onto $1 \%$ gelatine coated slides and coverslipped with phosphate-buffered glycerol ( $\mathrm{pH}$ 8.6).

For diaminobenzidine (DAB) processed sections, doublelabeling was performed on pairs of antigens that had localization in separate cellular compartments, i.e., nuclear (c-Jun) or cytoplasmic (ChAT or FG). The strategy was to first perform the processing for the antigen located in the nucleus, using nickel intensification to form a dark purple-black reaction product, then to complete the labeling for the cytoplasmic marker, using standard $\mathrm{DAB}$ processing to form a brown reaction product.

When the two primary antibodies of interest were raised in different host species, the following procedure was used. Free-floating sections were incubated in a $50 \%$ ethanol/3\% peroxidase solution to quench endogenous peroxidase activity, before a 30-min incubation in $0.1 \mathrm{M} \mathrm{PB} \mathrm{pH} 7.4$ containing 5\% NHS. Sections were then incubated for $48-72 \mathrm{~h}$ at room temperature with a mixture of $\mathrm{c}$-Jun (1:25,000; host rabbit) and ChAT (1:500; host goat) in PB containing $0.1 \%$ sodium azide, $2 \%$ NHS and $0.2 \%$ Triton X-100. After washes in $\mathrm{PB}$, sections were subsequently incubated overnight at room temperature with biotin-conjugated donkey anti-rabbit (1:1000). After washing in $\mathrm{PB}$, sections were incubated for $2 \mathrm{~h}$ in avidin-biotin complex (Vectastain ABC kit; Vector Laboratories, Burlingame, CA, 
USA), washed in $\mathrm{PB}$ and $0.1 \mathrm{M}$ acetate buffer $\mathrm{pH}$ 6.0, and incubated for $15 \mathrm{~min}$ in DAB solution $(0.2 \%$ D-glucose, $0.04 \%$ ammonium chloride, and $0.025 \%$ DAB in acetate buffer). Development of the reaction product was achieved by addition of $0.02 \%$ glucose oxidase, and a black reaction product was obtained by adding $2 \%$ nickel sulfate to the DAB solution. The development of reaction product was monitored by eye, and stopped by several washes in acetate buffer before a final wash in PB. The second antigen (ChAT) was then revealed by overnight incubation with biotin-conjugated donkey anti-goat (1:1000) and processed as described above but omitting nickel sulfate. After DAB processing, sections were mounted from $0.9 \%$ saline onto $1 \%$ gelatine coated slides, dried, and dehydrated through an ascending series of ethanols, followed by histolene, and coverslipped with DPX (Crown Scientific, Mulgrave, VIC, Australia). The procedure was modified when labeling c-Jun with FG, as both primary antisera were raised in the same species (rabbit). Here, the primary antisera were not mixed together but instead the entire procedure was completed for c-Jun staining alone (using nickel intensification), before incubating sections with anti-FG (1:2000) and completing the staining in the absence of nickel. Because FG is never found in the nucleus and c-Jun-immunoreactivity is exclusively nuclear, this allowed us to calculate the proportion of c-Junpositive retrogradely labeled neurons.

\section{ANTIBODIES}

Primary antibodies were obtained from the following sources (information on specificity has been provided by the manufacturers):

- Rabbit anti-c-Jun: polyclonal antibody generated against amino acids 1-79 of human c-Jun (Santa Cruz Biotechnology, Inc., Santa Cruz, CA, USA; sc-1694; batch F1203). Western blot analysis reveals a band at $\sim 43 \mathrm{kDa}$.

- Rabbit anti-ATF-3 (C-19): affinity purified polyclonal antibody generated against an ATF-3 C-terminal peptide of human origin (Santa Cruz Biotechnology, Inc., Santa Cruz, CA, USA; sc-188; batch J2209). Western blot analysis of RAW 264.7 whole cell lysate reveals a band at $\sim 27 \mathrm{kDa}$.

- Goat anti-ChAT: immunoaffinity purified polyclonal antibody generated against the human placental enzyme (Millipore, Temecula, CA, USA; AB144P; batch LV1450147). Western blot analysis of NIH/3T3 lysates reveals a band at $\sim 70 \mathrm{kDa}$, consistent with the predicted weight of ChAT protein.

- Rabbit anti-FG: polyclonal antibody generated against FG conjugated to bovine serum albumin (AbD Serotec, Kidlington, UK; 4512-4018; batch A950308).

Secondary antisera were raised in donkey and obtained from the following suppliers: anti-goat biotin-SP (Jackson Immunoresearch; 705-065-147; batch 51932), anti-rabbit biotin-SP (Jackson Immunoresearch; 711-065-152; batch 73931), anti-rabbit Cy3 (Jackson Immunoresearch; 711-165-152; batch 69532), anti-sheep AF488 (Invitrogen; A-11015; batch 84C1-1).

\section{NEURONAL COUNTS}

The prevalence of preganglionic neurons expressing each marker were calculated as a proportion of all positively labeled nucleated profiles of neurons within the entire extent of the intermediolateral column (IML; L1-L2, and L6-S1) and the central autonomic area (CAA; L1-L2). The following antibody combinations were assessed: c-Jun with ChAT, ATF-3 with ChAT, FG with ChAT, and FG with c-Jun. Double-labeled neurons with each marker were expressed as a percentage of all neurons of that chemical class. The number of sections available in a horizontal series was limited, therefore for each combination of antibodies and injury type, all immunolabeled neurons within the lumbar IML/CAA and sacral IML were recorded, averaging over 200 neurons per location/animal/injury.

\section{INTENSITY ANALYSIS}

To determine the relative intensity of ChAT immunostaining within preganglionic neurons, monochrome 8-bit images were collected at random along the IML within spinal levels L1-L2 and L6-S1 from sections processed either for fluorescent immunolabeling of FG with ChAT, or ATF-3 with ChAT (acquired using an Olympus BX51 microscope and RT Spot camera). Sections from the same animal were imaged using the same camera settings and exposure time. To ensure that bleaching of fluorophores did not influence the intensity of immunolabeling, the sections were exposed for the minimum length of time necessary and images for intensity analysis were collected before any other measurements or neuronal counts were performed. Intensity measurements were performed using Image-Pro Plus (Version 7; Media Cybernetics, Carlsbad, CA, USA) on images obtained using the $20 \times$ objective. Mean intensity of a single $6 \times 6$ pixel area of interest (equivalent to $4.3 \mu \mathrm{m}^{2}$ ) was measured within the brightest area of the cytoplasm of each neuron, from which an average value for each animal was calculated. Within the sacral IML, an average of 85 neurons per horizontal section were analyzed in each animal; within the lumbar IML around 60 neurons were analyzed per section per animal. In total, within the lumbar IML, 4226 neurons were analyzed in the nerve injury groups, and 842 neurons in naïve controls; within the sacral IML, 6535 neurons were analyzed in the nerve injury groups, and 1245 neurons in naïve controls.

\section{MEASUREMENTS OF NEURONAL SOMATA}

To determine the size of ChAT-positive preganglionic somata, monochrome 8-bit images were collected at random along the IML within lumbar L1-L2 and sacral L6-S1 from sections processed for DAB immunolabeling of c-Jun and ChAT (acquired using a Zeiss Axioimager M1 and MRm camera). Images were spatially calibrated from a reference image, and ChAT-positive neurons were traced using Image-Pro Plus. From each horizontal section, approximately 65 neurons were measured in the lumbar IML, and 110 in the sacral IML. In total, in the lumbar IML, 4035 neurons were measured in the nerve injury groups, and 464 in naïve controls; in the sacral IML, 6844 neurons were measured in the nerve injury groups, and 908 in naïve controls.

\section{STATISTICS AND FIGURE PREPARATION}

To detect neuronal changes ipsilateral to nerve injury (i.e., changes in proportions of neurons expressing a marker, fluorescence intensity or soma size), we performed a two-way ANOVA with repeated measures, with side (ipsilateral or contralateral) as the withinsubject factor and time as the between-subject factor. Dunnett's test was performed to determine if there was an effect of injury on the 
contralateral side relative to naïve controls. Numbers of FG-labeled neurons in sacral spinal cord ipsi- and contralateral to injury were compared using paired $t$-tests. The proportions of neurons expressing different markers were plotted as untransformed data but statistical analyses were performed following arcsine-root transformation. All statistical tests were performed using SPSS Statistics v. 19 (Chicago, IL, USA). Results are expressed as mean \pm SEM and $P<0.05$ was regarded as statistically significant.

For figure production, grayscale images of fluorescent samples were digitally colorized, and adjustments made where necessary in contrast and brightness to best represent the immunostaining as seen under the microscope (Adobe InDesign and Photoshop CS5; Adobe Systems, San Jose, CA, USA). Color images of DAB samples (acquired using an Olympus BX51 microscope and Zeiss HRc camera) were similarly adjusted in contrast and brightness to best represent the native immunostaining.

\section{RESULTS \\ A SUBPOPULATION OF AXOTOMIZED PREGANGLIONIC NEURONS UPREGULATED c-JUN EXPRESSION}

To locate neurons extending processes from the spinal cord into the abdomen, such as preganglionic neurons that innervate the major pelvic ganglion, we used the fluorescent tracer, FG (Anderson and Edwards, 1994). The distribution of retrogradely labeled FG-positive preganglionic neurons was comparable to previous descriptions in rat spinal cord (Figure 1; Hancock and Peveto, 1979a,b; Nadelhaft and Booth, 1984; Nadelhaft and McKenna, 1987; Strack et al., 1988). In lumbar spinal cord (L1-L2), FG neurons formed bilateral columns within the IML, and were also located within the CAA, between the dorsal corticospinal tract and central canal. The majority of sympathetic preganglionic neurons innervating the pelvic ganglion are located in the CAA (Hancock and Peveto, 1979b; Nadelhaft and McKenna, 1987; Strack et al., 1988), therefore after cutting the hypogastric nerve, we predicted that more axotomized neurons would be found here than the IML. In sacral spinal cord (L6-S1), FG neurons were found only within the IML, forming distinctive bilateral columns extending from the L5-L6 border to the most caudal aspect of S1. All parasympathetic preganglionic neurons innervating the pelvic ganglion are located here and, conversely, all sacral IML neurons project to the pelvic ganglion, therefore after cutting the pelvic nerve, we predicted that axotomized neurons would only be found in this region.

In naïve control rats, a low level of basal c-Jun expression was evident within both sacral and lumbar preganglionic neurons, and was restricted to neuronal nuclei. However, after nerve transection, a dramatic upregulation of c-Jun expression was detected (Figure 1). Statistical analyses showed that there was a significant main effect of side $(P<0.001)$ and an interaction between side and post-injury time $(P=0.034)$, but no effect of injury on the contralateral side relative to naïve controls (Figures 1A,C). The effect of injury was strongest in sacral cord ipsilateral to the site of pelvic nerve transection (Figures 1A-E), where approximately 55\% of FG neurons displayed upregulation of c-Jun expression 1 week after injury. Two and 4 weeks after injury, the proportion of FG neurons expressing c-Jun was reduced to approximately 30\% (Figure 1A). No upregulation of c-Jun above basal levels in sacral cord was detected after hypogastric nerve transection (Figure 1F).
We also counted all of the FG neurons in the sacral IML of horizontal cord sections, each section containing dozens of fluorescent FG neurons, ipsi- and contralateral to pelvic nerve injury. No evidence was found for neuronal cell death in this region, with comparable numbers of FG neurons being present ipsi- and contralateral to the injury, at all time points (ipsi vs. contra: 1 week, $160 \pm 28$ vs. $152 \pm 14 ; 2$ weeks, $205 \pm 39$ vs. $197 \pm 59 ; 4$ weeks, $232 \pm 25$ vs. $214 \pm 17 ; n=4$ rats).

After hypogastric nerve injury, there was no upregulation of c-Jun in the lumbar IML relative to basal levels (Figures 1G,L,M), but in the lumbar CAA c-Jun was increased at 1 week $(P=0.033$; Figures $\mathbf{1 H}, \mathbf{J}, \mathbf{N})$. The upregulation of c-Jun-positive neurons in the CAA compared with the IML is consistent with the former region providing greater input to the pelvic ganglion (Hancock and Peveto, 1979b; Nadelhaft and McKenna, 1987; Strack et al., 1988). Pelvic nerve transection had no significant effect on c-Jun expression in the lumbar CAA (Figures 1J,I) but did cause a small but significant upregulation of c-Jun in the lumbar IML ipsilateral to the injury (main effect of side, $P=0.034$ ) that showed an interaction with post-injury time $(P=0.026)$, being more prevalent at 1 week (Figure $1 \mathrm{~K}$ ).

Almost without exception, c-Jun-positive nuclei were restricted to FG neurons, indicating that c-Jun upregulation was specific to putatively injured preganglionic neurons rather than local interneurons. No glial expression of c-Jun was observed, in contrast to the profound upregulation of c-Jun after axotomy of pelvic ganglion neurons (Nangle and Keast, 2009).

Together, this set of experiments shows that upregulation of c-Jun is most pronounced in sacral spinal cord, ipsilateral to pelvic nerve injury. This upregulation is transient, and occurs in a subgroup of injured neurons. However, upregulation occurs in very few neurons that may be undergoing compensatory sprouting or aberrant growth, such as preganglionic neurons in other regions of the cord, or nearby spinal interneurons. We did not find evidence to support substantial death of neurons in the sacral IML after pelvic nerve injury, the scenario in which the greatest change in c-Jun expression occurred.

\section{AXOTOMY REDUCED ChAT EXPRESSION IN A SUBPOPULATION OF PREGANGLIONIC NEURONS}

To further define the characteristics of axotomized preganglionic neurons and examine properties specific to those neurons upregulating c-Jun expression, immunolabeling for c-Jun was performed in combination with ChAT, the synthetic enzyme for their primary neurotransmitter, acetylcholine. The prevalence and distribution of ChAT-positive neurons matched the location of FG-positive neurons and previous data (Barber et al., 1984). Representative micrographs illustrating the distribution of ChAT-positive neurons in sacral and lumbar cord 2 weeks after either pelvic or hypogastric nerve transection are shown in Figures 2A,B, respectively. Higher magnification images of ChAT and c-Jun immunoreactivity are provided in Figures 2C-F.

In naïve control spinal cord, c-Jun-positive neurons were scarce (see above and Figure 1) and comprised $<10 \%$ of ChAT-positive neurons in the sacral IML (Figure 2G), lumbar IML (Figure 2H), and lumbar CAA (Figure 2I). Conversely, in these three areas $>90 \%$ of all c-Jun-positive neurons were ChAT-positive (Figures 2J-L). 


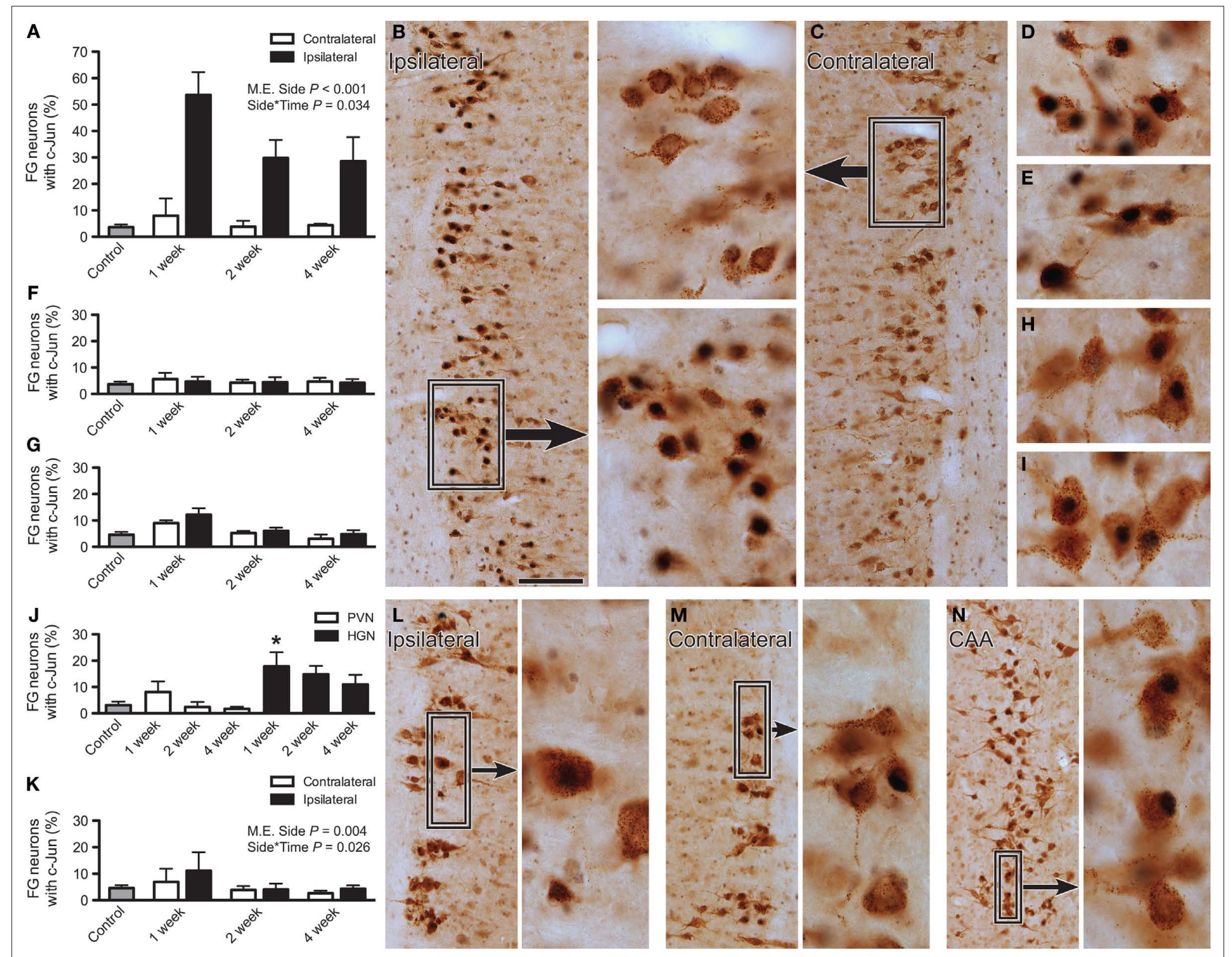

FIGURE 1 | Immunoreactivity for c-Jun in lumbar and sacral preganglionic neurons after axotomy. The proportion of FluoroGold (FG)-positive neurons expressing c-Jun was recorded in horizontal sacral (L6-S1) and lumbar (L1-L2) spinal cord sections 1, 2, or 4 weeks after nerve transection and in naïve controls. (A) In the sacral intermediolateral column (IML), the proportion of FG-positive neurons expressing c-Jun was significantly increased ipsilateral to injury (main effect of side: $F_{1,9}=159.78, P<0.001$ ) and there was also a significant interaction between side and time $\left(F_{2,9}=5.06, P=0.034\right)$, with the largest effect of injury apparent at 1 week. There was no effect contralateral to injury, when compared with naïve controls. (B) FG and c-Jun immunolabeling within the sacral IML, ipsilateral to pelvic nerve transection, 1 week after injury. Boxed region provides higher magnification images of c-Jun-positive nuclei within FG-positive neurons. (C) FG and c-Jun immunolabeling within the sacral IML, contralateral to pelvic nerve transection, 1 week after injury. (D,E) Higher magnification images of FG and c-Jun labeling in sacral IML, 2 and 4 weeks after pelvic nerve transection, respectively. $(\mathbf{F}, \mathbf{G})$ No effect of hypogastric nerve transection was detected in the sacral (F) or lumbar (G) IML, either in the context of side or relative to naïve controls. $(\mathbf{H}, \mathbf{I})$ Examples of $F G$ and c-Jun labeling in the central autonomic area (CAA), 2 and 4 weeks after hypogastric nerve transection, respectively. (J) There was a significant increase in proportion of FG neurons expressing c-Jun within the CAA after hypogastric (HGN) but not pelvic nerve (PVN) transection, with this effect occurring 1 week after injury; asterisk indicates a significant difference from naïve controls $\left(F_{6.27}=4.37\right.$,

$P=0.033)$. (K) There was a small but significant increase in the prevalence of c-Jun-positive FG neurons in the ipsilateral lumbar IML after pelvic nerve transection (main effect of side: $F_{19}=14.427, P=0.004$ ) and there was also a significant interaction between side and time $\left(F_{2,9}=5.664, P=0.026\right)$, with the largest effect of injury occurring at 1 week. There was no effect contralateral to injury, when compared with naive controls. (L) FG and c-Jun labeling within the lumbar IML, ipsilateral to hypogastric nerve transection, 1 week after injury; boxed region provides a higher magnification image to demonstrate a c-Jun-positive nucleus within a FG-positive neuron and an adjacent-Jun-negative neuron. (M) As per (L), contralateral to hypogastric nerve transection. (N) FG and c-Jun labeling within the CAA, 1 week after hypogastric nerve injury; boxed region shows c-Jun-positive nuclei within FG-positive neurons at higher magnification. Bars represent mean $\pm \mathrm{SEM}$, with $n=4$ for all groups. Main effects and interactions identified by two-way ANOVA (repeated measures) indicated on relevant plots. Comparisons with naïve controls made with Dunnett's test. Scale bar: $100 \mu \mathrm{m}$ (B,C), $40 \mu \mathrm{m}$ (boxes for B,C; D,E,H,I), $120 \mu \mathrm{m}$ (L,M), and $175 \mu \mathrm{m}$ (N); $30 \mu \mathrm{m}$ (boxes for $\mathbf{L}-\mathbf{N}$ ).
In parallel with our observations on FG neurons, after pelvic nerve injury there was a substantial increase in the proportion of ChAT-positive neurons expressing c-Jun in the ipsilateral side of the sacral IML $(P<0.001)$, which did not vary significantly with time $(P>0.05$; Figure 2G). Closer inspection of ChAT/c-Jun colocalization showed that in the sacral IML there was a significant main effect of side on prevalence of c-Jun-positive neurons that failed to express ChAT $(P=0.012$; Figures $2 \mathrm{C}, \mathbf{J})$. This occurred in 

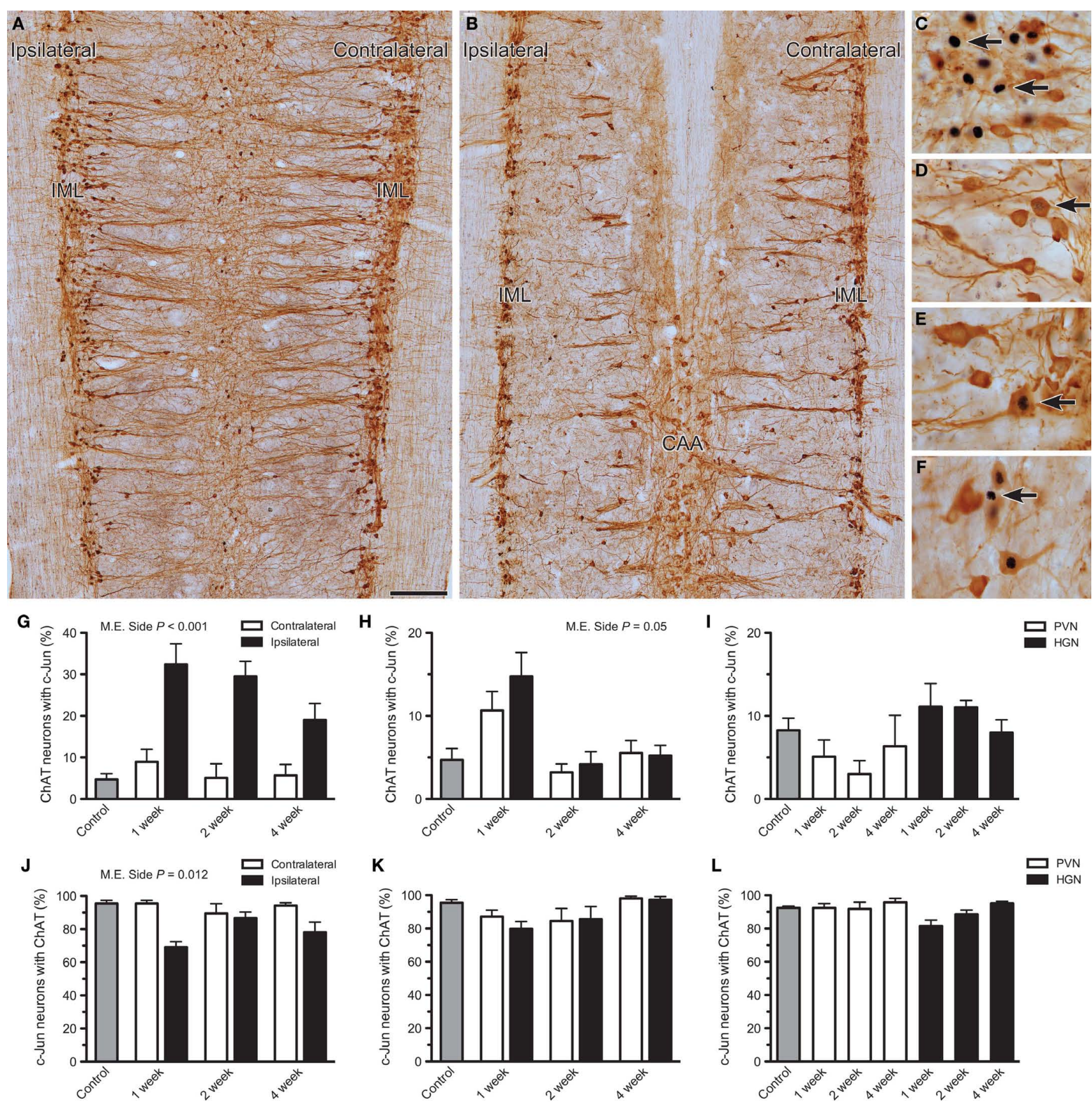

FIGURE 2 | Effects of axotomy on choline acetyltransferase (ChAT) immunoreactivity in preganglionic neurons. The proportion of c-Jun-positive neurons immunoreactive for ChAT was recorded in horizontal sacral (L6-S1) and lumbar (L1-L2) spinal cord sectionsone, 2, or 4 weeks after nerve transection and in naive controls. The proportion of ChAT-positive neurons expressing c-Jun was also analyzed. (A,B) Low power images illustrating the distribution of ChAT-positive neurons within sacral (A) and lumbar (B) cord, 2 weeks after pelvic or hypogastric nerve transection, respectively. (C,D) Higher magnification images showing c-Jun-positive nuclei in sacral intermediolateral column (IML), 1 week after pelvic nerve transection (C, ipsilateral; $\mathbf{D}$, contralateral). In (C), some of the neurons with c-Jun-positive nuclei are ChAT-negative (arrows). In (D), intense c-Jun immunoreactivity is absent, but some dim labeling is indicated (arrow). (E) A c-Jun-positive nucleus within a ChAT-positive neuron (arrow) in the lumbar IML contralateral to hypogastric nerve transection, 1 week after injury. (F) A c-Jun-positive nucleus within a ChAT-negative neuron (arrow) in the lumbar central autonomic area (CAA), 1 week after hypogastric nerve injury. (G) In the sacral IML, a significant increase in the proportion of ChAT neurons expressing c-Jun was detected ipsilateral to pelvic nerve transection (main effect of side: $F_{1,12}=94.144, P<0.001$ ) but there was no significant interaction between side and time. There was no effect contralateral to injury, when compared with naïve controls. (H) Ipsilateral to hypogastric nerve transection, the proportion of ChAT neurons expressing c-Jun in the lumbar IML may have been increased relative to the contralateral side, but this did not quite reach statistical significance (main effect of side: $F_{1,12}=4.75, P=0.05$ ). There was no significant effect contralateral to injury, when compared with naïve controls. (I) No consistent effect was detected within the CAA after either pelvic (PVN) or hypogastric (HGN) nerve transection. (J) A significant reduction in the proportion of c-Jun-positive neurons expressing ChAT was recorded in the sacral IML after pelvic nerve transection (main effect of side: $F_{1,12}=8.871, P=0.012$ ), but there was no interaction with time. There was no effect contralateral to injury, when compared with naïve controls. (K) There was no significant change in the proportion of c-Jun positive neurons expressing ChAT, ipsilateral to hypogastric nerve transection. There was no effect contralateral to injury, when compared with naïve controls. (L) No effect of PVN or HGN was detected in the CAA compared to naïve controls. Bars represent mean \pm SEM, with $n=5$ for all groups. Main effects identified by two-way ANOVA (repeated measures) indicated on relevant plots. Comparisons with naïve controls made with Dunnett's test. Scale bar: $200 \mu \mathrm{m}$ (A,B), $50 \mu \mathrm{m}$ (C-F). 
up to $30 \%$ of sacral IML neurons on the ipsilateral side. No loss of ChAT was noted in the scarce c-Jun-positive neurons contralateral to pelvic nerve injury in the sacral cord (Figures 2D,J).

One week after hypogastric nerve injury there appeared to be a small increase in c-Jun expression with ChAT neurons ipsilateral to injury (Figure $2 \mathrm{H}$ ), but this did not quite achieve statistical significance $(P=0.05)$. There was also no statistically significant effect of injury on the contralateral side or in the CAA, relative to naïve controls (Figures $\mathbf{2 H}, \mathbf{I}$ ). After hypogastric nerve injury there was no effect on the proportion of c-Jun-positive neurons expressing ChAT in the lumbar IML or CAA (Figures K,L).

Together, these results indicate that although c-Jun is persistently upregulated in a population of sacral preganglionic neurons after axotomy, a subgroup of these neurons undergoes profound downregulation of ChAT, such that during this period they would no longer be immunohistochemically classified as cholinergic. Therefore, we conducted an additional set of studies to quantify the preganglionic neurons where this has occurred. First, we quantified ChAT-negative neurons in the total population of preganglionic neurons identified by the retrograde tracer, FG. ChAT-negative FG neurons were rare in the lumbar and sacral IML of naïve controls (Figures 3A,B). However, ipsilateral to pelvic nerve injury, many FG neurons in the sacral IML no longer expressed ChAT (Figures 3A,D-F). This increased prevalence of the ChAT-negative population among the FG neurons on the ipsilateral side was statistically significant $(P<0.001)$ and varied significantly with time $(P=0.006)$, being greatest 1 week after injury. There was no effect of pelvic nerve transection on prevalence of ChAT-negative FG neurons on the contralateral side. Relative to naïve controls, an increase in the ChAT-negative population of FG neurons was also detected in both sides of the lumbar IML 1 week after hypogastric nerve transection (Figures 3B,G,H). This closely matched our observations on c-Jun expression (see above). In the CAA, the proportion of ChAT-negative FG neurons increased 1 week after hypogastric nerve transection $(P=0.033$; Figures 3C,I $)$.

Further characterization of preganglionic neurons that retained ChAT-immunoreactivity revealed a striking reduction in the intensity of immunolabeling, specific to sacral cord and limited to pelvic nerve transection (Figures $4 \mathrm{~A}-\mathrm{C}$ ). The intensity of ChATimmunofluorescence was significantly reduced on the ipsilateral side (Figures 4A,B; $P<0.001$ ). This reduction varied with time $(P=0.018)$, being greatest at 1 week. No significant changes were detected in the lumbar cord after either hypogastric or pelvic nerve injury, or in the sacral cord after hypogastric nerve injury (data not shown).

\section{SIZE OF ChAT-POSITIVE SACRAL PREGANGLIONIC NEURON SOMATA WAS SIGNIFICANTLY REDUCED AFTER PELVIC NERVE TRANSECTION}

To determine if axotomy caused structural changes in sacral IML neurons, images were collected from sections processed for ChAT immunolabeling (DAB method) and nucleated profiles of ChATpositive somata were measured. A significant decrease in soma size was detected after pelvic nerve injury (main effect of side, $P<0.001$ ) but this did not vary significantly with time. One week after pelvic nerve injury, ChAT-positive neurons were slightly but significantly smaller than naïve controls on both ipsilateral and contralateral sides (Figure 4D). No significant changes in soma size were found in the sacral IML after hypogastric nerve injury or in the lumbar IML after either nerve injury (data not shown). However it should be noted that within the lumbar IML, any effect is likely to be masked by uninjured neurons that do not project in the hypogastric nerve. The CAA was not examined in this set of experiments.

\section{ATF-3 WAS UPREGULATED IN AXOTOMIZED PREGANGLIONIC NEURONS}

We also examined the expression of ATF-3, another immediate early gene that is involved in responses to axonal injury (Herdegen et al., 1997; Takeda et al., 2000). In naïve controls and contralateral to either injury, no ATF-3 expression was detected within sacral or lumbar cord. However, after pelvic nerve transection, ChATpositive neurons expressing nuclear ATF-3 were recorded ipsilateral to injury (Figure 5A). This effect persisted over 4 weeks, during which approximately $10 \%$ of ChAT-positive neurons expressed ATF-3. ATF-3 expression was also observed in a smaller number of ChAT-positive neurons, both 1 and 2 weeks after hypogastric nerve transection, and both ipsilateral and contralateral to injury (Figure 5B). In the CAA, ATF-3 expression occurred in ChATpositive neurons, reaching approximately $5 \% 1$ week after injury and declining to approximately $2-3 \%$ by 2 and 4 weeks (Figure 5C). All ATF-3-positive nuclei were found within FG-positive somata and so were considered to be preganglionic neurons (Figures 5D-F). No glial expression of ATF-3 was observed in any spinal cord region.

In addition to expression of ATF- 3 in a minority of ChATpositive preganglionic neurons, there was a striking correlation between ATF-3-positive nuclei and ChAT-negative preganglionic neurons. This indicates ATF-3 is expressed preferentially in preganglionic neurons that undergo downregulation of ChAT. This effect was particularly pronounced in sacral IML after pelvic nerve transection, where 1 week after injury $65.3 \pm 4.5 \%$ of ATF-3-positive nuclei were within ChAT-negative neurons ( $n=4$ rats); the remaining ATF-3-positive nuclei were predominantly localized to weakly ChAT-positive neurons (Figure 5G). Two and 4 weeks after injury, the proportion of ATF-3-positive neurons that were ChAT-negative decreased to $40.3 \pm 1.6$ and $37.4 \pm 6.5 \%$, respectively ( $n=4$ rats). This relationship was also noted in lumbar cord after hypogastric nerve transection, albeit the observation was made bilaterally. That is, 1 week after injury, $51.1 \pm 7.6$ and $53.8 \pm 6.2 \%$ respectively of ATF-3 nuclei were found in ChAT-negative neurons in the ipsilateral and contralateral lumbar IML (Figure $5 \mathrm{H} ; n=4$ rats); by 2 and 4 weeks, almost all ATF-3 nuclei were within ChAT-positive neurons. In the CAA, $50.6 \pm 2.6,25.9 \pm 3.9$, and $20.7 \pm 8.9 \%$, respectively, of ATF-3 nuclei were found in ChAT-negative neurons at 1 , 2 , and 4 weeks after hypogastric nerve transection.

\section{DISCUSSION}

In this study we have shown that axotomy causes sacral preganglionic neurons to undergo rapid upregulation of immediate early gene (c-Jun, ATF-3) expression, and a decrease in ChAT expression and soma size. Many of these changes persist until at least 4 weeks after injury, in the context of a situation where regeneration has been prevented. Depending on the parameter measured, these structural and functional changes occurred in $50 \%$ or less of the total population of sacral preganglionic neurons, indicating that a subpopulation of neurons undergoes distinct degenerative changes. Whether this also indicates different potential for 

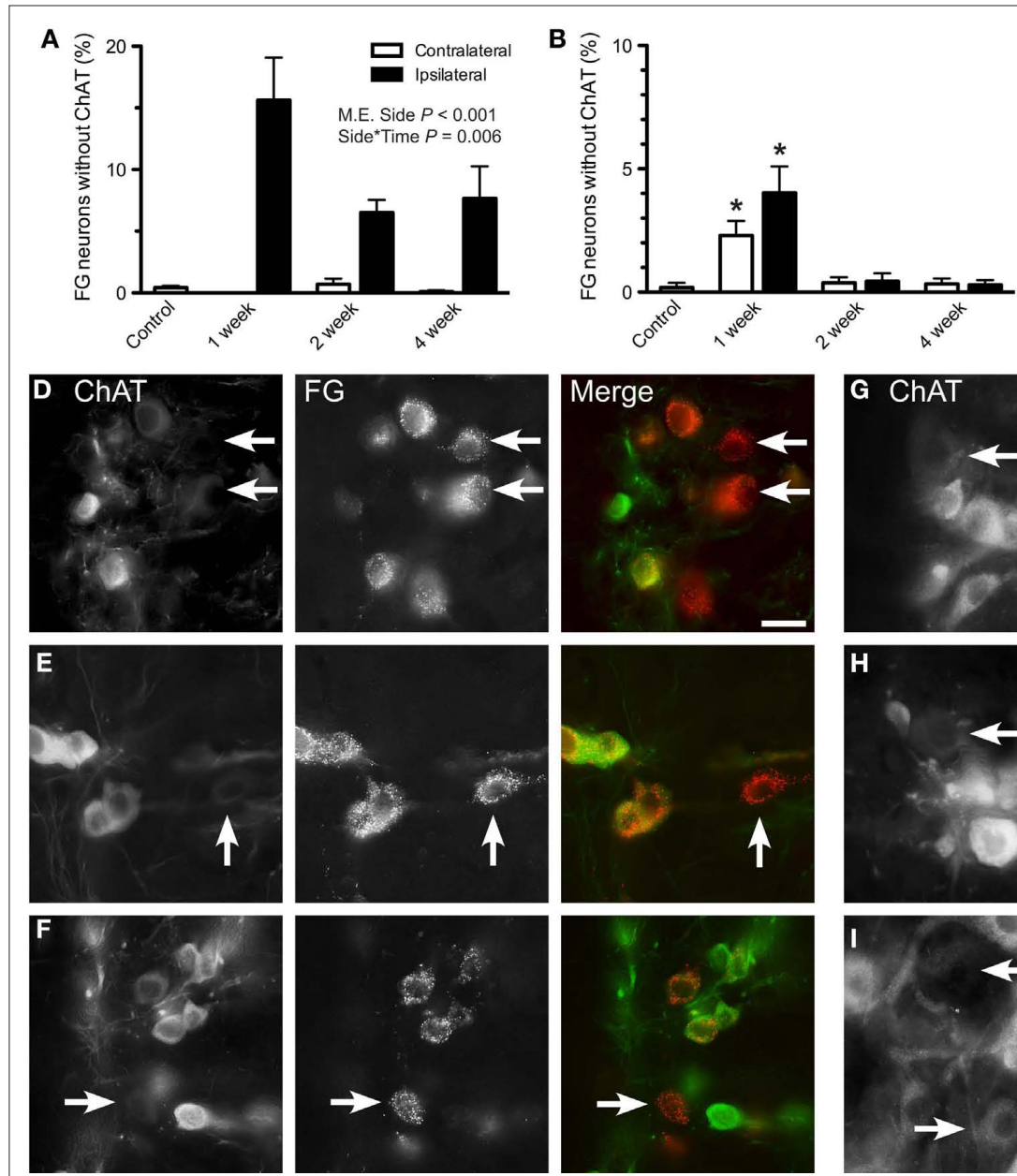
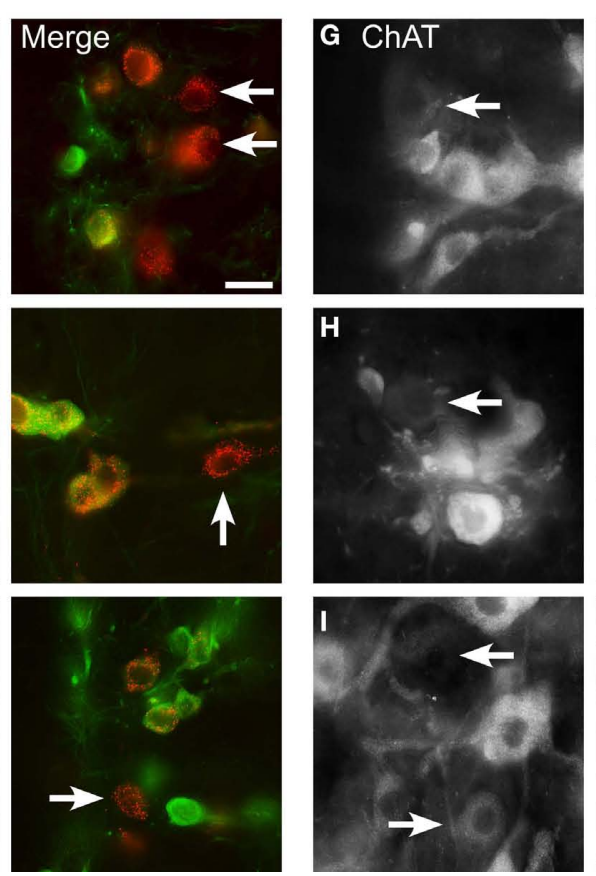
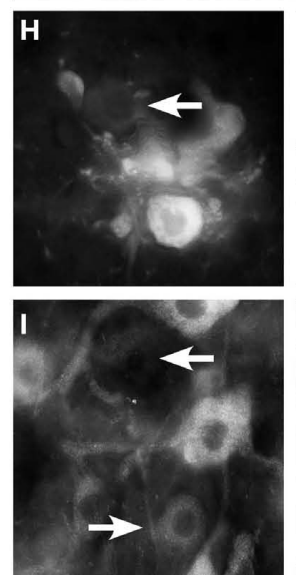
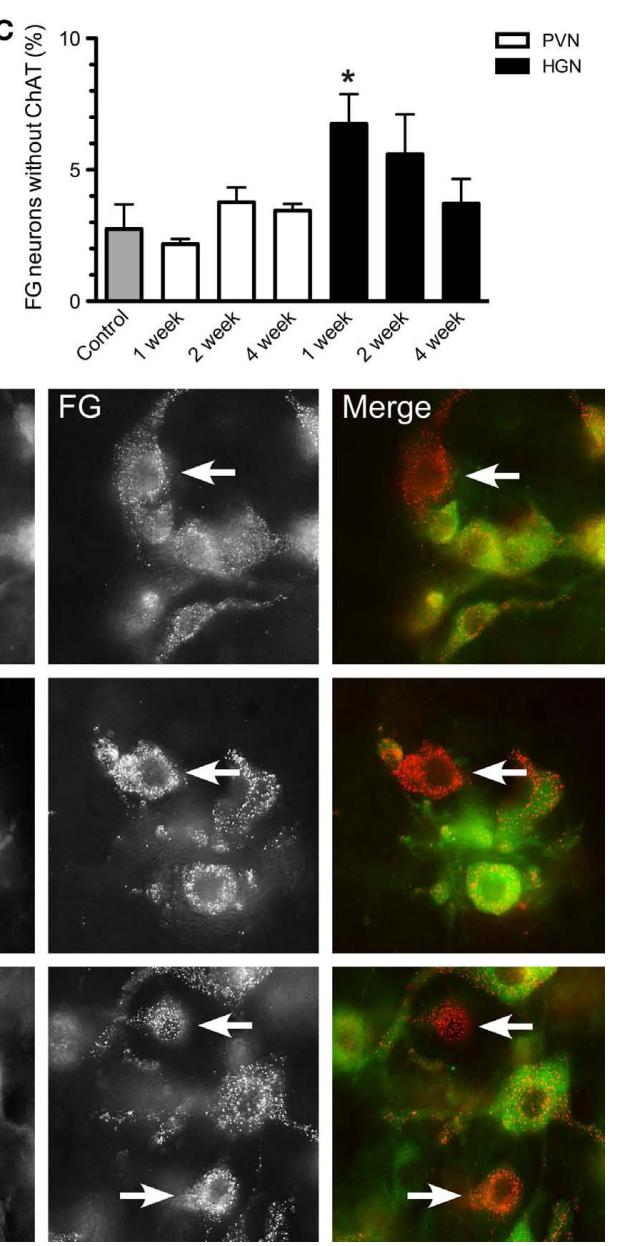

FIGURE 3 | Effects of axotomy on choline acetyltransferase (ChAT) immunoreactivity in retrogradely labeled preganglionic neurons. The proportion of FluoroGold (FG)-positive neurons with ChAT-immunoreactivity was recorded in horizontal sacral (L6-S1) and lumbar (L1-L2) spinal cord sections 1, 2 , or 4 weeks after nerve transection and in naïve controls. (A) In the sacral intermediolateral column (IML), the proportion of ChAT-negative FG neurons was significantly increased ipsilateral to pelvic nerve transection (main effect of side: $\left.F_{1,9}=194.87, P<0.001\right)$ and there was also a significant interaction between side and time $\left(F_{2,9}=9.521, P=0.006\right)$, with the largest effect of injury occurring at 1 week. There was no effect contralateral to injury, when compared with naïve controls. (B) Within the lumbar IML, there was no difference in the proportion of ChAT-negative FG neurons between sides, but relative to naïve controls there was an increase contralateral to injury at 1 week $\left(F_{3,15}=11.62\right.$, $P=0.007)$. Together this infers that there was also an increase in the ipsilateral at 1 week, relative to naïve controls. (C) One week after hypogastric nerve ( $H G N)$ transection, an increase in the proportion of ChAT-negative FG neurons was detected in the central autonomic area (CAA), compared to naïve controls $\left(F_{5,27}=2.3, P=0.033\right)$. No effect was seen at any other time or after pelvic nerve (PVN) transection. (D-F) Images of sacral IML ipsilateral to pelvic nerve transection, showing examples of ChAT-negative FG neurons at 1 (D), 2 (E), and 4 (F) weeks after injury. (G-I) Images of lumbar cord showing examples of ChAT-negative FG neurons, 1 week after hypogastric nerve transection, within the IML both ipsilateral $(\mathbf{G})$ and contralateral $(\mathbf{H})$ to injury, and within the CAA (I). Bars represent mean \pm SEM, with $n=4$ for all groups. Main effects and interactions identified by two-way ANOVA (repeated measures) indicated on relevant plots. Comparisons with naïve controls made with Dunnett's test. In B and $\mathrm{C}$, asterisk indicates a significant difference from naïve controls, with $P$ values as indicated above. Scale bar: $20 \mu \mathrm{m}$ (D-I). recovery of connectivity and function amongst distinct groups of parasympathetic neurons is yet to be established but may be informed by studies using incomplete injury (e.g., freezing or crushing pelvic nerves), where at least some regeneration would be expected (Keast, 2004).

We also observed upregulation of immediate early genes and loss of ChAT in lumbar spinal cord after hypogastric nerve injury. Generally these changes were more pronounced in the CAA than the IML, as predicted from the relative prevalence of pelvic ganglion-projecting neurons in each location. In contrast to the effects of unilateral pelvic nerve injury on sacral spinal neurons, we identified some bilateral effects on sympathetic preganglionic neurons after hypogastric nerve transection. This is supported by previous retrograde tracing studies to quantify preganglionic neurons extending axons along the hypogastric nerve (Hancock and Peveto, 1979b; Nadelhaft and McKenna, 1987). A limitation of our study was that we could not directly identify and quantify all sympathetic preganglionic neurons projecting in the hypogastric nerve (i.e., to the pelvic ganglion), which in these locations are likely to be outnumbered by preganglionic neurons supplying other targets. Retrograde labeling studies from the pelvic ganglion may provide additional insights into this particular group of sympathetic 

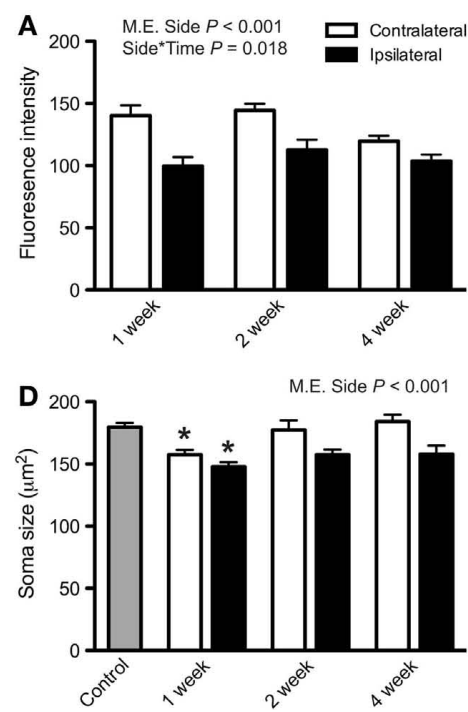
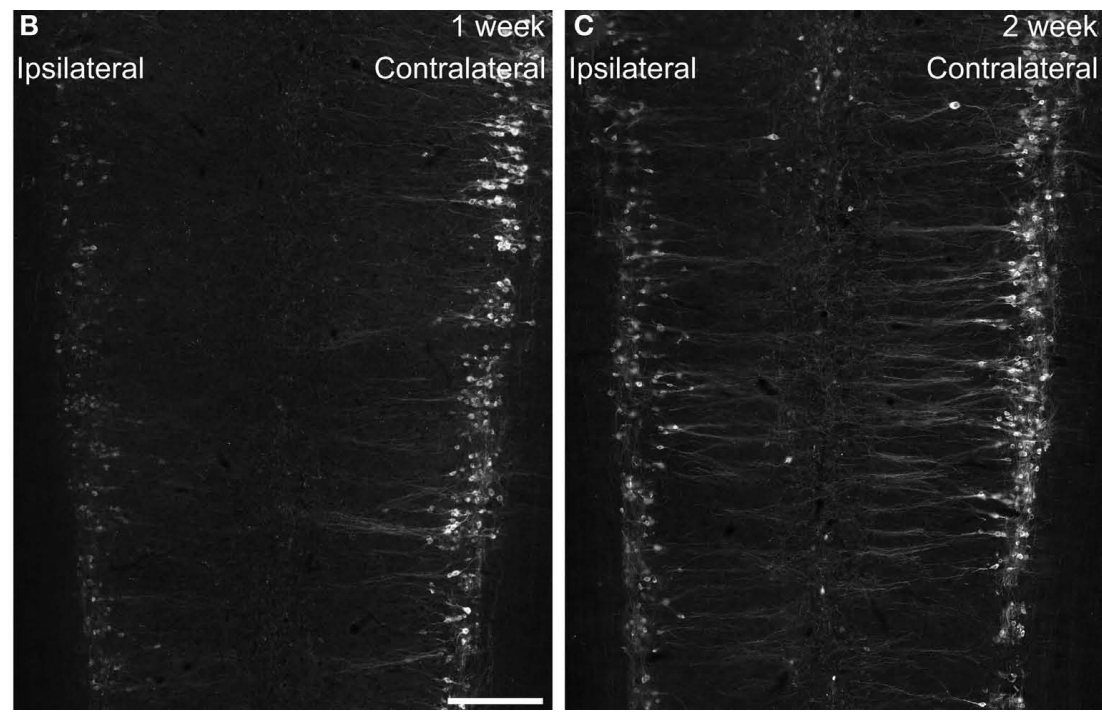

FIGURE 4 | Choline acetyltransferase (ChAT) immunoreactivity and soma size of sacral preganglionic neurons after pelvic nerve transection.

(A) Intensity of ChAT-immunofluorescence (0-256 grayscale, 8-bit images) in the sacral intermediolateral column (IML) was significantly reduced ipsilateral to pelvic nerve transection (main effect of side: $F_{1,19}=88.76, P<0.001$ ). There was also a significant interaction between side and time $\left(F_{2,19}=5.04\right.$, $P=0.018$, with the effect being largest at 1 week. (B,C) Image montages of sacral IML immunolabeled for ChAT, 1 (B) or 2 (C) weeks after pelvic nerve transection, each showing a reduction in labeling intensity ipsilateral to injury. (D) Area of soma profiles from ChAT-positive preganglionic neurons in the sacral IML were reduced ipsilateral to injury (main effect of side: $\left.F_{1,12}=37.922, P<0.001\right)$ but this was unaffected by time. One week after injury the somata on the contralateral side were slightly smaller than naïve controls $\left(F_{3,19}=4.853, P=0.025\right)$ and, because the ipsilateral neurons are smaller than contralateral neurons, the former are also smaller than naïve controls. Main effects and interactions identified by two-way ANOVA (repeated measures) indicated on relevant plots. Comparisons with naïve controls made with Dunnett's test. Bars represent mean \pm SEM, with $n=5$ for soma size analyses and 4-9 for intensity measurements. Scale bar: $300 \mu \mathrm{m}(\mathbf{B}, \mathbf{C})$. neurons. Irrespective, our observations do not suggest that injury has any more severe effect on sympathetic than parasympathetic pelvic ganglion-projecting preganglionic neurons, even though the former may be more susceptible to degenerative stimuli as they show a greater degree of aging-related atrophy (Dering et al., 1996; Santer et al., 2002).

Our results also contrast with previous studies of axotomy on more rostral populations of sympathetic preganglionic neurons where a substantial proportion of neurons (more than half) die after axotomy or treatment with antibodies against acetylcholinesterase (Tang and Brimijoin, 2002). We did not perform stereological analyses of lumbar or sacral preganglionic neurons, but in horizontal sections that each include a large sample of IML neurons, we did not see any decrease in FG-labeled neurons ipsilateral to injury; a previous study of unilateral pelvic nerve injury also failed to identify neuronal loss in the sacral IML, although it did report an upregulation of nitric oxide synthase (Vizzard et al., 1995). Therefore, while we cannot discount a small amount of neuronal death, it appears that the sympathetic preganglionic neurons injured in this study are more likely to survive after a transection injury. The type of injury performed may also impact on neuronal survival, because even though we did also not detect any neuronal loss in the sacral IML after pelvic nerve transection, 6 weeks after ventral root avulsion (an experimental model of cauda equina syndrome), almost $80 \%$ of preganglionic neurons degenerate (Hoang et al., 2003). It would be valuable to understand the cellular basis for avulsion being a more potent stimulus of neuronal death than transection in these neurons.
We observed a substantial reduction in ChAT expression in sacral parasympathetic preganglionic neurons after axotomy. A pronounced loss of ChAT expression has also been reported after avulsion injury of these neurons (Hoang et al., 2003) and in many other types of axotomized central neurons, but varies widely in the extent, duration and onset of loss so could not be predicted in our study (e.g., Hoover et al., 1985; Lams et al., 1988; Armstrong et al., 1991; Kou et al., 1995). Following pelvic nerve transection, the reduction in ChAT expression was so extreme in some neurons that they could no longer be categorized as cholinergic using typical immunohistochemical criteria (but could be identified as preganglionic and therefore originally cholinergic by presence of retrograde tracer). Apart from this practical demonstration that ChAT-immunoreactivity will underestimate the population of neurons surviving an injury, the results again reveal a functional change in a subpopulation (rather than all) of the preganglionic neurons. Why there should be such a broad spectrum of effects of axotomy (i.e., complete and partial loss of ChAT or no apparent change in ChAT) is interesting as it may again reflect differential susceptibility to injury or potential for successful regeneration.

Numerous studies have been performed on the expression of the transcription factor, c-Jun, after injury, when it is strongly correlated with the "cell body response" (as reviewed by Herdegen and Leah, 1998). This comprises a complex array of structural, chemical, and electrophysiological changes by which the neuron adapts for survival after injury, and in many cases upregulates genes necessary for regeneration. Axotomy-induced expression of c-Jun varies widely in latency and persistence, depending on neuron type, 
A

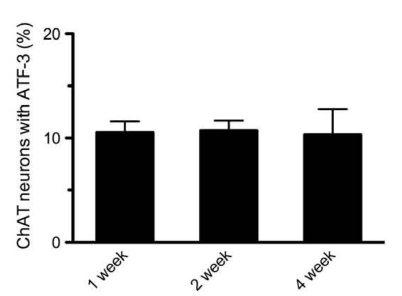

B

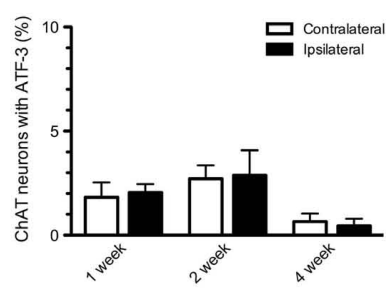

C
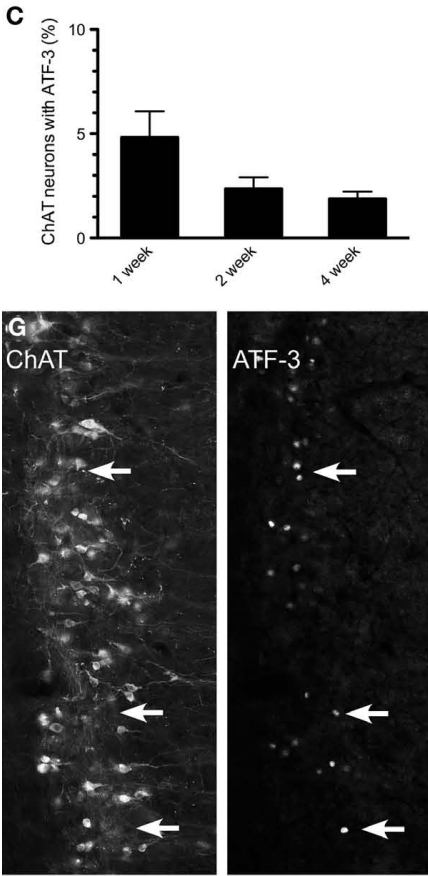

H

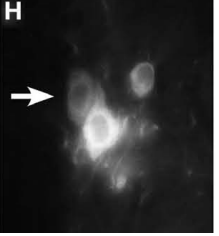

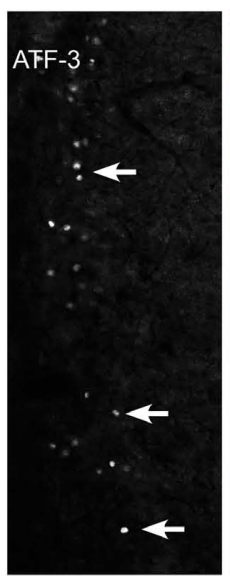

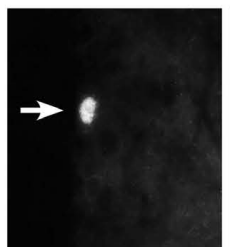

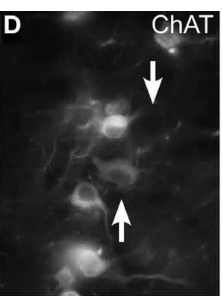
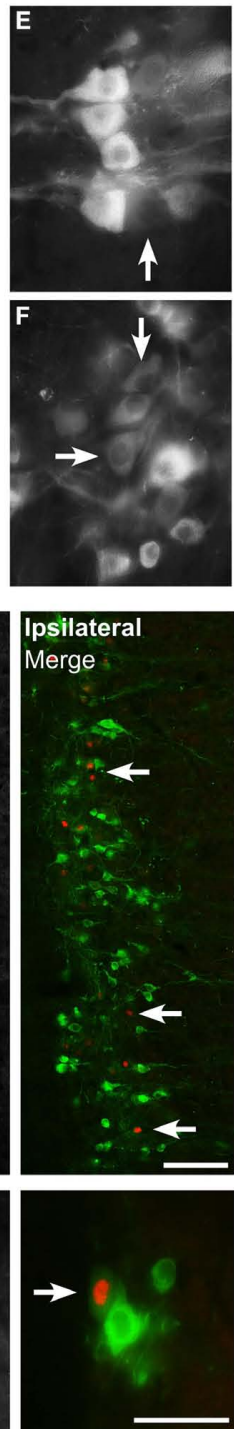

FIGURE 5 | Activating transcription factor-3 expression in axotomized lumbar and sacral preganglionic neurons. The proportion of choline acetyltransferase (ChAT)-positive neurons expressing activating transcription factor-3 (ATF-3) was recorded in horizontal sacral (L6-S1) and lumbar (L1-L2) spinal cord sections at 1,2, and 4 weeks after nerve transection and in naïve controls. (A) In the sacral intermediolateral column (IML), some ChAT-positive neurons expressed ATF-3 after pelvic nerve transection; no ATF-3 expression was detected contralateral to nerve injury at any time point, or in naïve controls. (B) In the lumbar IML a small proportion of ChAT-positive neurons expressed ATF-3 after hypogastric nerve transection; this was detected bilaterally but there was no difference between sides. No ATF- 3 was detected in lumbar IML of naïve controls or after pelvic nerve transection. (C) In the central autonomic area (CAA), a minority of ChAT-positive neurons expressed ATF-3 after hypogastric nerve transection, peaking at 1 week, and declining over the following weeks. No ATF-3
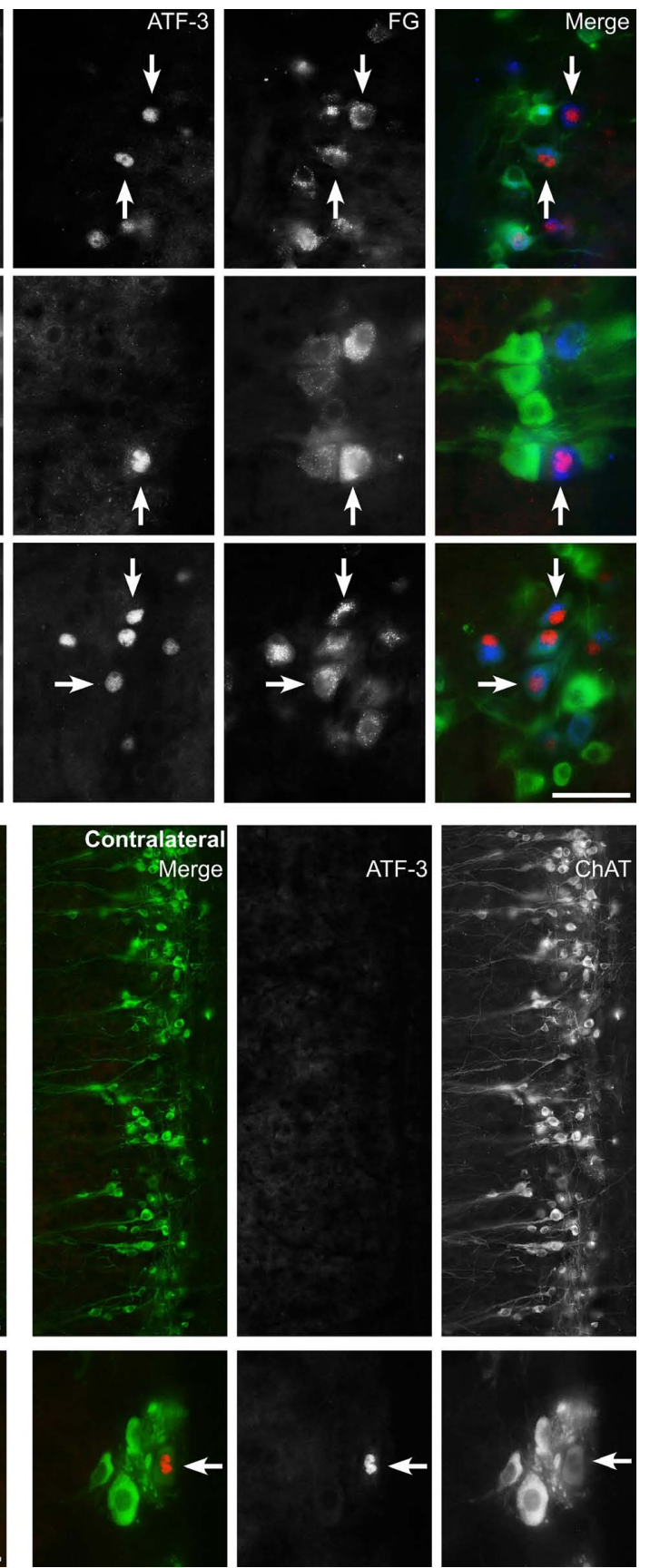

expression was detected within the CAA in naïve controls or after pelvic nerve transection. (D-F) All ATF-3-positive nuclei were found within FluoroGold (FG)-positive neurons, with representative images from sacral (D) and lumbar IML (E) and CAA (F) horizontal spinal cord sections 1 week after injury. Arrows highlight ATF-3-positive FG neurons across each series. (G) Ipsilateral and contralateral sides from the same horizontal section of sacral spinal cord labeled for ChAT and ATF-3, 1 week after pelvic nerve transection. ATF-3-positive nuclei are located in the IML ipsilateral to injury, and are almost exclusively within weakly ChAT-positive, or ChAT-negative neurons. The contralateral side displays no ATF-3 immunoreactivity. (H) Both ipsilateral and contralateral to a hypogastric nerve transection, examples of ATF-3 immunoreactivity in weakly ChAT-positive neurons. Potential changes in the ipsilateral side were examined by two-way ANOVA (repeated measures). Bars represent mean \pm SEM, with $n=4$ for all groups. Scale bars: $50 \mu \mathrm{m}$ (D-F), $100 \mu \mathrm{m}$ (G), and $50 \mu \mathrm{m}$ (H). 
with onset ranging from 5 to $36 \mathrm{~h}$, most commonly reaching a maximum in 3-5 days and potentially persisting up to 100 days. We also observed elevated c-Jun up to 4 weeks after axotomy but did not determine the time of onset of the response ( 1 week after injury was our earliest time point). An elegant study by Raivich et al. (2004) showed that c-Jun is required for facial nerve regeneration in mice; moreover, downregulation of c-Jun can occur if regeneration is achieved (Herdegen et al., 1991; Leah et al., 1991; Jenkins et al., 1993). Our experimental approach precluded regeneration (a segment of nerve was removed), and c-Jun remained elevated up to 4 weeks after transection, but it is possible that this was not sustained further, even though regeneration did not occur.

Activating transcription factor-3 has been implicated in similar injury-related events and is typically regarded as a cellular marker of stress and/or injury, which may follow the activation of c-Jun (Hai et al., 1999; Takeda et al., 2000; Tsujino et al., 2000; Raivich and Behrens, 2006). The functional consequences of ATF-3 induction are not well understood. ATF-3 may also play a role in determining cell fate after injury by directing the cell toward an apoptotic or regenerative response (Nakagomi et al., 2003; Song et al., 2008) and ATF-3 expression may promote neuron survival (Herdegen et al., 1997). What regulates the functional variability, and context dependency of c-Jun and ATF-3 actions is not yet well understood, although exposure to trophic factors can influence the relative expression of each transcription factor (Vaudano et al., 2001; Wang et al., 2003; Wu et al., 2003; Averill et al., 2004; Jongen et al., 2005; Parsadanian et al., 2006; Pezet et al., 2006; Hyatt Sachs et al., 2007; Young et al., 2008). A number of neurotrophic factors have been implicated in development or maintenance of sympathetic preganglionic neurons (Schober and Unsicker, 2001), but with the exception of a small number of preganglionic neurons in the sacral cord that express the receptor for GDNF (Forrest and Keast, 2008), the trophic factors for pelvic ganglion-projecting lumbosacral preganglionic neurons are unknown. In our study, we prevented regeneration of preganglionic neurons, so in this context availability of neurotrophic factors during the four postoperative weeks may not have altered. However, transecting these nerves also directly injures the peripheral processes of sensory fibers from relevant lumbar and sacral dorsal root ganglia, so we cannot predict what impact this may have had on the chemistry and activity of the spinal cord of those segments.

Our results from quantitation of c-Jun and ATF-3 in the spinal cord clearly indicate responses in a specific subgroup (rather than all) injured neurons. As well as the issues discussed earlier regarding the biological basis of this distinction, this particular aspect of our study raises the possibility of co-activation in the same group of injured neurons, consistent with their ability to co-dimerize (Lindwall and Kanje, 2005). This co-activation has been demonstrated elsewhere in the nervous system (Takeda et al., 2000; Nakagomi et al., 2003; Song et al., 2008). Because both primary antibodies were raised in the same species, we were unable to conduct double-labeling studies to directly visualize c-Jun and ATF-3 in the same neurons, however our observations in relation to ChAT expression indicate that injured neurons that have strongly downregulated ChAT expression (i.e., FG neurons that no longer express ChAT), may express both c-Jun and ATF-3, although it is possible that the former slightly predominates.
One of the primary aims of our study was to determine if uninjured neurons undergo plasticity, specifically axonal sprouting, following injury to the alternative source of preganglionic neurons to pelvic ganglia. This has been proposed by in vivo physiological studies where the pelvic or hypogastric nerves were injured (Dail et al., 1989; de Groat and Kawatani, 1989; Kihara et al., 1996). The issue is important because this type of growth could form the basis of alternative regenerative approaches to restore some types of central control or, may even need to be attenuated if aberrant growth leads to inappropriate hyperactivity states. Because we have previously shown that c-Jun was upregulated in sprouting, deafferented pelvic ganglion neurons and their adjacent glia (Nangle and Keast, 2009), we considered that c-Jun expression patterns may identify uninjured sprouting neurons in the current experiments. However, we found little evidence for increased c-Jun expression in the lumbar spinal cord after pelvic (sacral) nerve injury, with only a very small, transient increase in prevalence of c-Jun-positive neurons in the lumbar IML and no change in the CAA. We also found no expression in the sacral spinal cord after hypogastric nerve injury, and no glial expression of this immediate early gene in lumbar or sacral preganglionic neurons after either injury. While our observations do not support the proposal of collateral growth from a substantial number of uninjured preganglionic neurons, it is possible that sprouting is occurring but not coupled to c-Jun expression. In order to definitively demonstrate the cellular basis of plasticity observed in previous in vivo measurements of reflex function after injury, it may be necessary to seek aberrant synapses in isolated pelvic ganglia using intracellular electrophysiological recording approaches, or to perform anterograde labeling studies from spinal nuclei following injury. Our failure to observe expression of c-Jun in glia closely associated with somata of axotomized preganglionic neurons contrasts with the extensive c-Jun upregulation in glia within pelvic ganglia following degeneration of preganglionic axons (Nangle and Keast, 2009). This could be due to distinct physiological roles of central and peripheral glia, or different stimuli for c-Jun activation arising in the environment of degenerating axons or abnormally functioning deafferented pelvic ganglion neurons.

\section{CONCLUSION}

Transection of the pelvic or hypogastric nerves caused a range of potentially degenerative changes in injured preganglionic neurons, although there was no evidence of neuronal death. Because each of the effects we observed occurred in only a subpopulation of preganglionic neurons at that spinal level, this raises the possibility that distinct functional subgroups have different susceptibility to trauma-induced degeneration and potentially different regenerative abilities. The cellular mechanisms underpinning these distinct changes may provide new strategies for promoting regeneration of specific pathways within pelvic autonomic nerve circuits after surgically induced injury or spinal nerve trauma due to other causes.

\section{ACKNOWLEDGMENTS}

This work was supported by the National Health and Medical Research Council (Australia) Project Grant \#512140 and Senior Research Fellowships \#358709 and \#632903 to Janet R. Keast. 


\section{REFERENCES}

Anderson, C. R., and Edwards, S. L. (1994). Intraperitoneal injections of Fluorogold reliably labels all sympathetic preganglionic neurons in the rat. J. Neurosci. Methods 53, 137-141.

Armstrong, D. M., Brady, R., Hersh, L. B., Hayes, R. C., and Wiley, R. G. (1991). Expression of choline acetyltransferase and nerve growth factor receptor within hypoglossal motoneurons following nerve injury. J. Comp. Neurol. 304, 596-607.

Averill, S., Michael, G. J., Shortland, P. J., Leavesley, R. C., King, V. R., Bradbury, E. J., McMahon, S. B., and Priestley, J. V. (2004). NGF and GDNF ameliorate the increase in ATF3 expression which occurs in dorsal root ganglion cells in response to peripheral nerve injury. Eur. J. Neurosci. 19, 1437-1445.

Barber, R. P., Phelps, P. E., Houser, C. R., Crawford, G. D., Salvaterra, P. M., and Vaughn, J. E. (1984). The morphology and distribution of neurons containing choline acetyltransferase in the adult rat spinal cord: an immunocytochemical study. J. Comp. Neurol. 229, 329-346.

Dail, W. G., and Evan, A. P. (1978). Effects of chronic deafferentation on adrenergic ganglion cells and small intensely fluorescent cells. J. Neurocytol. 7 , 25-37.

Dail, W. G., Galindo, R., Leyba, L., and Barba,V.(1997).Denervation-induced changes in perineuronal plexuses in the major pelvic ganglion of the rat: immunohistochemistry for vasoactive intestinal polypeptide and tyrosine hydroxylase and histochemistry for NADPH-diaphorase. Cell Tissue Res. 287, 315-324.

Dail, W. G., Walton, G., and Olmsted, M. P. (1989). Penile erection in the rat: stimulation of the hypogastric nerve elicits increases in penile pressure after chronic interruption of the sacral parasympathetic outflow. J. Auton. Nerv. Syst. 28, 251-257.

de Groat, W.C., and Kawatani, M. (1989). Reorganization of sympathetic preganglionic connections in cat bladder ganglia following parasympathetic denervation. J. Physiol. 409, 431-449.

Dering, M. A., Santer, R. M., and Watson, A. H. (1996). Age-related changes in the morphology of preganglionic neurons projecting to the rat hypogastric ganglion. J. Neurocytol. 25, 555-563.

Forrest, S. L., and Keast, J. R. (2008). Expression of receptors for glial cell line-derived neurotrophic factor family ligands in sacral spinal cord reveals separate targets of pelvic afferent fibers. J. Comp. Neurol. 506, 989-1002.

Fowler, C. J. (1999). Neurological disorders of micturition and their treatment. Brain 122(Pt 7), 1213-1231.
Hai, T., Wolfgang, C. D., Marsee, D. K., Allen, A. E., and Sivaprasad, U. (1999). ATF3 and stress responses. Gene Expr. 7, 321-335.

Hancock, M. B., and Peveto, C.A. (1979a). A preganglionic autonomic nucleus in the dorsal gray commissure of the lumbar spinal cord of the rat. J. Comp. Neurol. 183, 65-72.

Hancock, M. B., and Peveto, C.A. (1979b). Preganglionic neurons in the sacral spinal cord of the rat: an HRP study. Neurosci. Lett. 11, 1-5.

Herdegen, T., Kummer, W., Fiallos, C. E., Leah, J., and Bravo, R. (1991). Expression of c-JUN, JUN B and JUN D proteins in rat nervous system following transection of vagus nerve and cervical sympathetic trunk. Neuroscience 45, 413-422.

Herdegen, T., and Leah, J. D. (1998). Inducible and constitutive transcription factors in the mammalian nervous system: control of gene expression by Jun, Fos and Krox, and CREB/ATF proteins. Brain Res. Rev. 28, 370-490.

Herdegen, T., Skene, P., and Bahr, M. (1997). The c-Jun transcription factor - bipotential mediator of neuronal death, survival and regeneration. Trends Neurosci. 20, 227-231.

Hoang, T. X., Nieto, J. H., Tillakaratne, N. J., and Havton, L. A. (2003). Autonomic and motor neuron death is progressive and parallel in a lumbosacral ventral root avulsion model of cauda equina injury. J. Comp. Neurol. 467, 477-486.

Hoover, D. B.,Hancock, J.C., and DePorter, T. E. (1985). Effect of vagotomy on cholinergic parameters in nuclei of rat medulla oblongata. Brain Res. Bull. 15, 5-11.

Hyatt Sachs, H., Schreiber, R. C., Shoemaker, S. E., Sabe, A., Reed, E., and Zigmond, R. E. (2007). Activating transcription factor 3 induction in sympathetic neurons after axotomy: response to decreased neurotrophin availability. Neuroscience 150, 887-897.

Jenkins, R., McMahon, S. B., Bond, A. B., and Hunt, S. P. (1993). Expression of c-Jun as a response to dorsal root and peripheral nerve section in damaged and adjacent intact primary sensory neurons in the rat. Eur. J. Neurosci. 5, 751-759.

Jongen, J. L., Haasdijk, E. D., SabelGoedknegt, H., van der Burg, J., Vecht, C. J., and Holstege, J. C. (2005). Intrathecal injection of GDNF and BDNF induces immediate early gene expression in rat spinal dorsal horn. Exp. Neurol. 194, 255-266.

Keast,J.R.(1995).Visualization and immunohistochemical characterization of sympathetic and parasympathetic neurons in the male rat major pelvic ganglion. Neuroscience 66, 655-662.

Keast, J. R. (1999). Unusual autonomic ganglia: connections, chemistry, and plasticity of pelvic ganglia. Int. Rev. Cytol. 193, 1-69.

Keast, J. R. (2004). Remodelling of connections in pelvic ganglia after hypogastric nerve crush. Neuroscience $126,405-414$.

Kenney, A. M., and Kocsis, J. D. (1998), Peripheral axotomy induces long-term c-Jun amino-terminal kinase-1 activation and activator protein-1 binding activity by c-Jun and junD in adult rat dorsal root ganglia in vivo. J. Neurosci. 18, 1318-1328.

Kepper, M. E., and Keast, J. R. (1998). Specific targeting of ganglion cell sprouts provides an additional mechanism for restoring peripheral motor circuits in pelvic ganglia after spinal nerve damage. J. Neurosci. 18 7987-7995.

Kihara, H., Kakizaki, H., and de Groat, W. C. (1996). Reorganization of the innervation of the vas deferens after sympathetic decentralization. Am. J. Physiol. 271, R1481-R1488.

Kou, S. Y., Chiu, A. Y., and Patterson, P. H. (1995). Differential regulation of motor neuron survival and choline acetyltransferase expression following axotomy. J. Neurobiol. 27, 561-572.

Lams, B. E., Isacson, O., and Sofroniew, M. V. (1988). Loss of transmitterassociated enzyme staining following axotomy does not indicate death of brainstem cholinergic neurons. Brain Res. 475, 401-406.

Leah, J. D., Herdegen, T., and Bravo, R. (1991). Selective expression of Jun proteins following axotomy and axonal transport block in peripheral nerves in the rat: evidence for a role in the regeneration process. Brain Res. 566, 198-207.

Lindwall, C., and Kanje, M. (2005). The Janus role of c-Jun: cell death versus survival and regeneration of neonatal sympathetic and sensory neurons. Exp. Neurol. 196, 184-194.

Nadelhaft, I., and Booth, A. M. (1984). The location and morphology of preganglionic neurons and the distribution of visceral afferents from the rat pelvic nerve: a horseradish peroxidase study. J. Comp. Neurol. 226, 238-245.

Nadelhaft, I., and McKenna, K. E. (1987). Sexual dimorphism in sympathetic preganglionic neurons of the rat hypogastric nerve. J. Comp. Neurol. 256, 308-315.

Nakagomi, S., Suzuki, Y., Namikawa, K., Kiryu-Seo, S., and Kiyama, H. (2003). Expression of the activating transcription factor 3 prevents c-Jun $\mathrm{N}$-terminal kinase-induced neuronal death by promoting heat shock protein 27 expression and Akt activation. J. Neurosci. 23, 5187-5196.

Nangle, M. R., and Keast, J. R. (2009). Deafferentation and axotomy each cause neurturin-independent upregulation of c-Jun in rodent pelvic ganglia. Exp. Neurol. 215, 271-280.

Orendacova, J., Cizkova, D., Kafka, J., Lukacova, N., Marsala, M., Sulla, I., Marsala, J., and Katsube, N. (2001). Cauda equina syndrome. Prog. Neurobiol. 64, 613-637.

Parsadanian, A., Pan, Y., Li, W., Myckatyn, T. M., and Brakefield, D. (2006) Astrocyte-derived transgene GDNF promotes complete and long-term survival of adult facial motoneurons following avulsion and differentially regulates the expression of transcription factors of AP- 1 and ATF/CREB families. Exp. Neurol. 200, 26-37.

Pezet, S., Krzyzanowska, A., Wong, L. F., Grist, J., Mazarakis, N.D., Georgievska, B., and McMahon, S. B. (2006). Reversal of neurochemical changes and pain-related behavior in a model of neuropathic pain using modified lentiviral vectors expressing GDNF. Mol. Ther. 13, 1101-1109.

Podnar, S., Trsinar, B., and Vodusek, D. B. (2006). Bladder dysfunction in patients with cauda equina lesions. Neurourol. Urodyn. 25, 23-31.

Purinton, P. T., Fletcher, T. F., and Bradley, W. E. (1973). Gross and light microscopic features of the pelvic plexus in the rat. Anat. Rec. 175, 697-706.

Raivich, G. (2008). c-Jun expression, activation and function in neural cell death, inflammation and repair. $J$. Neurochem. 107, 898-906.

Raivich, G., and Behrens, A. (2006). Role of the AP-1 transcription factor c-Jun in developing, adult and injured brain. Prog. Neurobiol. 78, 347-363.

Raivich, G., Bohatschek, M., Da Costa, C., Iwata, O., Galiano, M., Hristova, M., Nateri,A.S., Makwana, M., Riera-Sans, L., Wolfer, D. P., Lipp, H. P., Aguzzi, A., Wagner, E. F., and Behrens, A. (2004). The AP-1 transcription factor c-Jun is required for efficient axonal regeneration. Neuron 43, 57-67.

Samson, G., and Cardenas, D. D. (2007). Neurogenic bladder in spinal cord injury. Phys. Med. Rehabil. Clin. N. Am. 18, 255-274, vi.

Santer, R. M., Dering, M. A., Ranson, R. N., Waboso, H. N., and Watson, A. H. (2002). Differential susceptibility to ageing of rat preganglionic neurones projecting to the major pelvic ganglion and of their afferent inputs. Auton. Neurosci. 96, 73-81.

Schober, A., and Unsicker, K. (2001). Growth and neurotrophic factors regulating development and 
maintenance of sympathetic preganglionic neurons. Int. Rev. Cytol. 205, 37-76.

Song, D.Y., Yang, Y.C., Shin, D. H., Sugama, S., Kim, Y.S., Lee, B. H., Joh, T. H., and Cho, B. P. (2008). Axotomy-induced dopaminergic neurodegeneration is accompanied with c-Jun phosphorylation and activation transcription factor 3 expression. Exp. Neurol. 209, 268-278.

Strack, A. M., Sawyer, W. B., Marubio, L. M., and Loewy, A. D. (1988). Spinal origin of sympathetic preganglionic neurons in the rat. Brain Res. 455, 187-191.

Tabatai, M., Booth, A. M., and de Groat, W.C. (1986). Morphological and electrophysiological properties of pelvic ganglion cells in the rat. Brain Res. 382, 61-70.

Takeda, M., Kato, H., Takamiya, A., Yoshida, A., and Kiyama, H. (2000). Injury-specific expression of activating transcription factor- 3 in retinal ganglion cells and its colocalized expression with phosphorylated c-Jun. Invest. Ophthalmol. Vis. Sci. 41, 2412-2421.

Tang, H., and Brimijoin, S. (2002). Death of preganglionic sympathetic neurons after surgical or immunologic lesion of peripheral processes. Exp. Neurol. 177, 105-114.

Tsujino, H., Kondon, E., Fukuoka, T., Dai, Y., Tokunaga, A., Miki, K., Yonenobu, K., Ochi, T., and Noguchi, K. (2000). Activating transcription factor 3 (ATF3) induction by axotomy in sensory and motoneurons: a novel neuronal marker of nerve injury. Mol. Cell. Neurosci. 15, 170-182.

Vaudano, E., Rosenblad, C., and Bjorklund, A. (2001). Injury induced c-Jun expression and phosphorylation in the dopaminergic nigral neurons of the rat: correlation with neuronal death and modulation by glial-cellline-derived neurotrophic factor. Eur. J. Neurosci. 13, 1-14.

Vizzard,M.A.,Erdman, S.L., and de Groat, W. C. (1995). Increased expression of neuronal nitric oxide synthase (NOS) in visceral neurons after nerve injury. J. Neurosci. 15, 4033-4045.

Wang, R., Guo, W., Ossipov, M. H., Vanderah, T. W., Porreca, F., and Lai, J. (2003). Glial cell line-derived neurotrophic factor normalizes neurochemical changes in injured dorsal root ganglion neurons and prevents the expression of experimental neuropathic pain. Neuroscience 121, 815-824.

Wu, W., Li, L., Yick, L. W., Chai, H., Xie, Y., Yang, Y., Prevette, D. M., and Oppenheim, R. W. (2003). GDNF and BDNF alter the expression of neuronal NOS, c-Jun, and p75 and prevent motoneuron death following spinal root avulsion in adult rats. $J$. Neurotrauma 20, 603-612.

Young, B. A., Girard, B. M., and Parsons, R. L. (2008). Neurturin suppresses injury-induced neuronal activating transcription factor 3 expression in cultured guinea pig cardiac ganglia. $J$. Comp. Neurol. 508, 795-805.
Conflict of Interest Statement: The authors declare that the research was conducted in the absence of any commercial or financial relationships that could be construed as a potential conflict of interest.

Received: 17 December 2010; accepted: 07 January 2011; published online: 25 January 2011.

Citation: Peddie CJ and Keast JR (2011) Pelvic nerve injury causes a rapid decrease in expression of choline acetyltransferase and upregulation of c-Jun and ATF-3 in a distinct population of sacral preganglionic neurons. Front. Neurosci. 5:6. doi: 10.3389/ fnins.2011.00006

This article was submitted to Frontiers in Autonomic Neuroscience, a specialty of Frontiers in Neuroscience.

Copyright $\odot 2011$ Peddie and Keast. This is an open-access article subject to an exclusive license agreement between the authors and Frontiers Media SA, which permits unrestricted use, distribution, and reproduction in any medium, provided the original authors and source are credited. 